\title{
El apoyo familiar en el proceso de integración educativa de estudiantes con necesidades educativas en condición de discapacidad
}

\author{
Angélica Fontana Hernández. \\ Académica de la División de Educación Básica \\ Centro de Investigación y Docencia en Educación, Universidad Nacional \\ Heredia, Costa Rica \\ Ana Laura Alvarado Valverde ${ }^{2}$ \\ Docente en la Escuela Eduardo José Pinto Hernández \\ Alajuela, Costa Rica \\ Mauren Angulo Ramírez ${ }^{3}$ \\ Docente en la Escuela Guadalupe de Alajuela \\ Alajuela, Costa Rica \\ Elizabeth Marín Valverde \\ Docente en la Escuela Rincón Grande de Pavas \\ San José, Costa Rica \\ Dania Melissa Quirós Salas ${ }^{5}$ \\ Docente en la Escuela Rincón de Cacao \\ Alajuela, Costa Rica
}

Recibido 25 de mayo de 2009 • Aceptado 14 de setiembre de 2009

${ }^{\mathrm{T}}$ Bachiller en Problemas de Aprendizaje, Licenciada en Educación Especial con énfasis en Retardo Mental y Máster en Pedagogía con énfasis en la diversidad de procesos educativos de la Universidad Nacional. Académica de la División de Educación Básica del CIDE y responsable del Proyecto UNA Educación de Calidad, que atiende estudiante con necesidades educativas en su formación profesional. Tiene experiencia en educación primaria y en los servicios de Educación Especial. Tiene publicaciones en el campo de la pedagogía.

Correo electrónico: angelfontana@racsa.co.cr

${ }^{2}$ Licenciada en Educación Especial con énfasis en integración. Actualmente se desempeña como docente en el servicio de Apoyo Fijo en Problemas de Aprendizaje en la escuela Eduardo José Pinto Hernández.

Correo electrónico: jojo0616@hotmail.es

\footnotetext{
${ }^{3}$ Licenciada en Educación Especial con énfasis en integración. Actualmente se desempeña como docente en el servicio de Apoyo de Problemas de Aprendizaje en la escuela Guadalupe.

Correo electrónico: maufa81@yahoo.es

${ }^{4}$ Licenciada en Educación Especial con énfasis en integración. Actualmente se desempeña como docente en el servicio de Apoyo Fijo en Retardo Mental en la escuela Rincón Grande de Pavas.

Correo electrónico: elecita@costarricense.cr

${ }^{5}$ Licenciada en Educación Especial con énfasis en integración. Actualmente se desempeña como docente en el servicio de Apoyo Fijo en Problemas Emocionales y de Conducta en la escuela Rincón de Cacao.

Correo electrónico: daniqusa09@hotmail.com
} 
Resumen. En el proceso de socialización, la familia tiene un papel fundamental en el cuidado y la crianza de los hijos y, principalmente, en la transmisión de conocimientos, valores y costumbres que les permita adaptarse a la sociedad como personas activas y productivas. En los últimos años, el sistema educativo costarricense ha experimentado cambios significativos, debido a los procesos de integración escolar de estudiantes que presentan necesidades educativas, porque requieren de diferentes apoyos y recursos especializados para su formación y su desarrollo integral. Por lo anterior, se generó la inquietud de investigar sobre el apoyo familiar en el proceso de integración escolar de estudiantes a los cuales se les aplica adecuación curricular significativa en una escuela pública y, de esta forma, determinar los tipos de apoyo que proporcionan los miembros de la familia, así como la forma en que estos apoyos favorecen el proceso de integración escolar.

Palabras clave. Apoyo familiar, proceso de integración escolar y estudiantes con necesidades educativas.

Abstract. In the process of socialization, the family has a fundamental role in the care and raising of the children, but principally, in the transmission of knowledge, values and customs that allow them to adapt to the society as active and productive individuals. In the last years, the Costa Rican educational system has experienced significant changes, due to the processes of students' school integration, who present educational needs, because they need specialized supports and different resources for their formation and integral development. Thus, a concern was generated so that the familiar support is investigating in the process of students' school integration, who are the ones who receive significant curricular adaptations in the public schools, and for instance, it was necessary to determine the kind of supports that are provided to the members of the family, as well as, the ways in which these supports can benefit the process of school integration.

Key words. Family support, processes of students' school integration and educational needs.

\section{A manera de introducción}

En los fríos amaneceres, cuando los tibios rayos del sol calientan y la hora de entrada a clases se acerca, se aprecia cientos de estudiantes que se dirigen a sus escuelas, muchos tomados de la mano de un familiar, y otros que se acercan solitariamente, al portón principal, para hacer notar su asistencia. Cada uno de ellos lleva en su rostro sonrisas, alegres y sobre sus hombros no sólo la carga escolar, sino también, la esperanza de formar un fututo mejor. Detrás de ellos, y con similares sueños, metas y anhelos está su núcleo familiar, brindando apoyo para que todas estas ilusiones se conviertan en realidad.

En el proceso de socialización la familia tiene un papel fundamental en el cuidado y la crianza de los hijos y, principalmente, en la transmisión de conocimientos, valores y costumbres, así como, en el desarrollo de las habilidades emocionales y sociales que facilitan su adaptación para integrarse a la sociedad como personas activas y productivas.

Es por esto que diversas corrientes tanto psicológicas, sociológicas como educativas, afirman que la familia es la base de la sociedad, a la cual se le asigna el deber de velar por el bienestar de todos sus miembros y de satisfacer sus necesidades económicas, sociales, emocionales y educativas.

En el ámbito educativo, la familia debe preocuparse por brindar todo el apoyo posible para que la formación de sus hijos se lleve a cabo de la mejor manera, especialmente, en el proceso de integración educativa de estudiantes que presentan necesidades educativas en condición de discapacidad, ya que es en el interior de esta estructura social, en donde se construyen las bases lingüísticas, preceptuales, emocionales y sociales necesarias para desarrollar destrezas, adquirir nuevos conocimientos y formar valores en otros contextos. 
Por tanto, la familia es un núcleo social y el primer sistema de formación del individuo (Campabadal, 2001) que influye, de manera particular, en las interrelaciones con las otras estructuras sociales que conforman la sociedad (la comunidad, la iglesia, la escuela entre otras), ya que se transforma, constantemente, dadas sus características internas de variabilidad y adaptabilidad, así como por las condiciones socioculturales (económicas, educativas, geográficas, lingüísticas, entre otras) en que se encuentran inmersas.

Por lo anterior, la conformación de las familias está determinada tanto por las condiciones de su origen, como por las interrelaciones y los cambios producidos en las diferentes etapas de su evolución histórica. Sin embargo, un aspecto fundamental, es la existencia de los vínculos afectivos que une a cada uno de sus miembros; ésta es la característica esencial que define a la familia, actualmente, en la sociedad global.

Según Guang, Campos y Smith, Caparrós (citados por Campabadal, 2001) se pueden identificar las siguientes etapas en el desarrollo histórico de la familia:

1. Unión por promiscuidad sexual, la cual da origen a la conformación de la familia. Las interrelaciones entre los seres humanos se centran en la búsqueda de sentimientos de seguridad, de afecto y de protección.

2. Promoción de relaciones grupales, denominada matrimonio en grupos, en los cuales, se creaban relaciones de unión entre un grupo de hombres y un grupo de mujeres, lo que dio origen a la poliginia debido a que era permitido la relación de un hombre con varias mujeres y, al mismo tiempo, se practicaba la poliandria, es decir, una mujer con varios hombres a la vez.

3. Evolución de la unión y surgimiento de la familia consanguínea, en la cual, se prohibía la unión sexual con los progenitores. Sin embargo, esta restricción no limitaba la unión entre hermanos.

4. Surgimiento de la familia punalúa o de compañeros íntimos. Se constituyeron los matrimonios por grupos, es decir, un conjunto de hombres y mujeres se unían y establecían vínculo de parejas. En esta etapa, los hijos pertenecían de la madre y ya prevalecía la prohibición de las relaciones entre hermanos.

5. Emersión de la familia sindiásmica, en la cual el hombre vivía solamente con una mujer, no obstante, contaba con el derecho de ser infiel. En esta etapa, la mujer debía mantener una posición de fidelidad, de lo contrario era duramente castigada. La separación era permitida, y si ésta se daba, la madre era quien quedaba con el cuido de sus hijos.

6. Aparición de la familia patriarcal, en la que se dio la pérdida del poder de la mujer y se estableció el derecho hereditario paterno, en el cual, el hombre era el jefe de la familia y podía vivir en poligamia. El resto del grupo (la mujer y los hijos) se sometían a su autoridad y dominio.

7. Constitución de la familia monogámica, en la cual, se aseguró la paternidad indiscutible de los hijos y su derecho hereditario. Se afianzó la unión entre el hombre y la mujer, y se torna más difícil la separación. Se considera que la monogamia se fundamenta más en aspectos económicos que afectivos.

Se puede afirmar, entonces, que a través de la historia han existido diferentes formas de unión entre los seres humanos, las que, al final, dieron origen a la familia actual, la cual, se ha conformado de diferentes formas, según las características del contexto social y económico de cada época histórica. 
Por otra parte, considerando los cambios estructurales que ha sufrido la familiar se pueden establecer los siguientes tipos de familia:

Familia nuclear. Es la forma más común de vida familiar y se compone de los padres, pero no necesariamente casados, y sus hijos. Ésta funciona y reside, por lo general, en forma independiente de otras unidades familiares con las que puede estar emparentada.

Familia extensa. Se compone de dos o más familias nucleares y diversos parientes, que viven juntos en la misma casa, en las mismas tierras o muy próximos unos de otros.

Familia mixta especial. Está conformada por el padre, madre y los hijos de ambos.

Familia uniparental. Es la forma que más se ha difundido en la actualidad y se compone de un solo progenitor y uno o más hijos (Flores y Rodríguez, 2000).

Es importante resaltar, que los cambios producidos al interior de las estructuras de las familias generan modificaciones no sólo en las interrelaciones personales y sociales, sino, también, en la conceptuación de lo que es una familia, pasando desde una visión tradicional (unión de padres biológicos y sus hijos) a una perspectiva más amplia, en la cual se incluye en el núcleo familiar a otros miembros, según sus necesidades socioeconómicas y contextos en que se desarrollan.

Esta constante renovación y modificación de la estructura familiar da origen al concepto de familia que se utiliza en este trabajo, propuesto por Arés (1997a) quien expresa que la "familia es la unidad social constituida por un grupo de personas unidas por vínculos consanguíneos, afectivos y/o cohabitacionales que llenan necesidades en el ser humano para su desarrollo integral" (p. 16).

De acuerdo con la anterior definición, se considera que la familia puede estar conformada por cualquier persona que atienda, apoye y guíe el desarrollo vital de cada uno de sus miembros, sin centrarse solamente en el concepto tradicional, que incluye padre, madre y hermanos, porque, como se ha mencionado hasta el momento, las modificaciones a las estructuras sociales son constantes, lo que implica una transformación en cuanto a la apreciación y a la conceptuación del entorno familiar.

Por tanto, la familia es el ambiente idóneo para que los miembros que la conforman adquieran las habilidades individuales y sociales necesarias para su integración personal y social. Al respecto, Arés (1997b) menciona que la familia tiene diversas funciones, entre ellas se encuentran: procrear nuevos individuos para la sociedad, proporcionarles cuido y protección, construir nuevas formas de comunicación e intercambio de afecto, propiciar la estructuración de la identidad en cada uno de sus miembros de acuerdo con las características de cada etapa de desarrollo, sus intereses y sus necesidades y promover diversos espacios de recreación para el disfrute del ocio y las atracciones culturales y naturales.

Además, una función relevante que debe cumplir la familia y que se destaca en este trabajo es la educativa, porque el núcleo familiar es el primer medio de formación para sus miembros, ya que propicia la adquisición de conceptos, valores, actitudes y destrezas fundamentales para que los individuos se integren y se adapten a la sociedad en la que se desenvuelven.

Si se parte de la premisa de que la Educación Básica (Preescolar, I, II y III ciclos) es esencial para la adquisición de nuevos conceptos y el despliegue de las habilidades necesarias en los niveles cognoscitivo, social, afectivo y psicomotor, cobra importancia la idea de considerar el apoyo familiar ${ }^{6}$ como un aspecto determinante en los procesos de enseñanza y de aprendizaje de todos los estudiantes, especialmente, de aquellos que presentan necesidades educativas ${ }^{7}$.

\footnotetext{
${ }^{6}$ Son aquellas acciones que realizan uno o varios miembros del contexto familiar, con el fin de colaborar en la formación educativa del estudiante. En éste se toman en cuenta los siguientes aspectos: económicos, sociales, académicos y emocionales (elaboración del grupo de investigación).

${ }^{7}$ Son las condiciones de aprendizaje que surgen de la interacción entre las características personales y sociales de los estudiantes y el entorno social que presenta barreras para el aprendizaje, tales como: actitudinales, pedagógicas, arquitectónicas y culturales (elaboración del grupo de investigación).
} 
Dada la importancia de desarrollar diversas acciones que promuevan la participación y el acceso de todas las personas, a los servicios educativos en igualdad de condiciones y oportunidades, desde el año 1997 se han realizado en el país diferentes investigaciones con el objetivo de conocer, comprender y analizar el proceso de integración de estudiantes con necesidades educativas en condición de discapacidad en el sistema educativo costarricense.

Al respecto, la Organización Panamericana de la Salud (2004) expresa:

El reto que asume la educación costarricense es propiciar la inclusión en mayores espacios de participación de una manera técnicamente adecuada y sostenida, en la cual la formación tanto de los docentes como del personal administrativo, desempeña un papel predominante para garantizar el respeto de los derechos de acceso a la educación de las niñas, niños, adolescentes y adultos con discapacidad. (p. 39)

Es por esto que la educación actual enfrenta el reto de responder a las exigencias de la diversidad estudiantil y de continuar con su función socializadora. Los y las docentes, especialmente, tienen la responsabilidad de proporcionar diversas respuestas didácticas y apoyos a las necesidades educativas que presentan los y las estudiantes, y, a la vez, continuar atendiendo las exigencias curriculares para el resto del estudiantado.

En este momento, el grupo de estudiantes que requiere de apoyo educativo para acceder a la educación ha aumentado considerablemente, y se considera que este sistema de compensación constituye el medio, por el cual los estudiantes que presentan necesidades educativas asociadas a una discapacidad o a sus diferencias individuales, pueden acceder a una formación educativa en igualdad de oportunidades, lo cual, va a promover su integración social y laboral.

El país cuenta con legislación que garantiza estos apoyos en el sistema educativo; tenemos la Ley 7600: Igualdad de oportunidades para las personas con discapacidades del año 1996, su Reglamento, de 1998 y Políticas, normativa y procedimientos para el acceso a la Educación de estudiantes con necesidades educativas, de 1997. Más recientemente, la Ley 8661: Convención sobre los derechos de las personas con discapacidad y su protocolo, de 2008.

Por lo anterior, se generó la inquietud de investigar sobre el apoyo familiar en el proceso de integración educativa de estudiantes con necesidades educativas en condición de discapacidad, a quienes se les aplica una adecuación curricular significativa en una escuela pública de San José $\mathrm{y}$, con ello, analizar el tipo de ayuda que proporcionan los miembros de la familia; determinar la coordinación que se presenta entre los docentes de I y II ciclos, la docente de Educación Especial y los miembros de la familia en el establecimiento del apoyo educativo que requieren los estudiantes, así como la forma en que esa ayuda favorece el proceso de integración educativa.

El contar con estudios que aborden esta temática puede brindar información valiosa a los docentes sobre la importancia del apoyo familiar en el proceso de integración educativa de estudiantes con necesidades educativas en condición de discapacidad, con el fin de contribuir al mejoramiento de la calidad de la educación que se proporciona a esta población estudiantil.

Así pues, el presente estudio sirve de soporte a la práctica profesional de los docentes de las carreras de Educación Especial y de Pedagogía en I y II ciclos, pues permite un acercamiento a la temática desde la perspectiva de los padres y estudiantes. Otro aporte significativo consiste en brindar datos relevantes al Ministerio de Educación Pública sobre el impacto del apoyo familiar en el proceso de integración escolar de estudiantes con necesidades educativas, con el fin de generar acciones desde las Asesorías de Educación Especial de las Direcciones Regionales para que promuevan la participación de los padres en el contexto educativo. 
En el ámbito educativo, la integración educativa se concibe como un asunto de justicia social, sobre todo, cuando se trata de los derechos humanos que tienen las personas con necesidades educativas en condición de discapacidad, a educarse en un ambiente lo menos restringido posible, lo que asegura, por su parte, una participación social y laboral más comprometida.

La integración educativa hace énfasis en el principio de normalización, sectorización e individualización, los cuales pretenden brindar a las personas con necesidades educativas en condición de discapacidad los recursos y el apoyo necesarios, con base en la valorización de sus características personales y sociales, en la elaboración de planeamientos individuales y en la aplicación de las adecuaciones curriculares y adecuaciones de acceso, así como, en la creación de servicios de apoyo en Educación Especial.

De acuerdo con el currículo oficial, éstas son las principales estrategias que utiliza este modelo de integración educativa, con la finalidad de que todos los estudiantes puedan obtener las metas que se proponen en el ámbito educativo.

Este modelo integracionista generó cambios significativos en las propuestas pedagógicas en Costa Rica, ya que los docentes y los administrativos de los centros educativos requerían desarrollar mayores competencias profesionales para ajustar las estrategias didácticas, los recursos y los materiales a las necesidades educativas que manifiestan los estudiantes.

Por otra parte, en este estudio, al referirse al apoyo familiar, se torna necesario establecer algunas vinculaciones entre el núcleo con quien viven día a día los estudiantes y el ámbito educativo, de tal forma que éste se refleje en el proceso de formación que se desarrolla, particularmente, en el proceso de integración educativa.

Se entiende por apoyo familiar aquellas acciones que realizan uno o varios miembros del contexto familiar (desde la perspectiva del concepto de familia utilizado en este documento), que favorecen los procesos de enseñanza y de aprendizaje del estudiante con necesidades educativas en condición de discapacidad, considerando las características personales y sociales.

Desde esta visión, se les presenta a los padres o encargados de los estudiantes con necesidades educativas la oportunidad de participar en el proceso educativo de sus hijos, tal como lo establece la Ley 7600 (Costa Rica, Asamblea Legislativa, 1996) en su artículo 20, en el que se garantiza a los padres el derecho a participar en la selección, ubicación, organización y evaluación de los servicios educativos. De este modo, se resalta la importancia de la permanencia o intervención constante de los miembros de la familia en el proceso educativo.

Al respecto, Heward (1997) considera que las relaciones entre la familia y el entorno escolar promueven el acceso a una amplia variedad de actividades y de esfuerzos sociales enfocados hacia los comportamientos significativos mostrados por el estudiante fuera del contexto escolar, y hacia la aplicación de diversas estrategias en el hogar, para generar un mayor rendimiento académico.

Se puede entender, de esta forma, la importancia de la intervención de la familia en el proceso educativo que se sigue, ya que este núcleo se encuentra en constante contacto con el estudiante, como lo mencionan Giangreco, Clininger e Iverson (citados por Heward, 1997, p. 508).

La familia conoce mejor que nadie las características del niño.

Las familias son las que tienen mayor interés en que sus hijos aprendan.

Es muy probable que la familia sea el único grupo que va a intervenir en la educación del niño a lo largo de toda su educación...

Las familias pueden influir positivamente en la calidad de los servicios educativos que se ofrecen en la comunidad.

Las familias deberán vivir todos y cada uno de los días con los resultados de las decisiones que tomen los equipos educativos. 
Se puede afirmar que es mediante la coordinación entre los ámbitos familiar y educativo, que se pueden cumplir las metas que se proponen para el desarrollo personal y social de las personas con necesidades educativas especiales.

Es importante resaltar que cada uno de los ambientes familiares ofrece diferentes tipos de apoyo a sus hijos, de acuerdo con las características particulares que lo conforman, con el objetivo de satisfacer las necesidades que cada estudiante presenta. Este apoyo puede verse influido por factores tanto internos (conformación familiar, educación de sus miembros y recursos económicos) como externos (ubicación geográfica, medios de comunicación y servicios de la comunidad), los cuales constituyen variables específicas en la crianza y la formación de los hijos.

Por lo anterior, es necesario que el núcleo familiar intervenga en la formación educativa de los hijos y establezca una comunicación constante con el centro educativo, para coordinar y sobrellevar cualquier situación que emerja en alguno de los dos contextos. Igualmente, debe existir una relación con los miembros de la comunidad que faciliten la participación social y laboral.

Es importante destacar que, en el ámbito nacional, los documentos que tratan sobre las normas y los procedimientos de los servicios de Educación Especial, establecen ciertas pautas para la participación de los padres en la educación de sus hijos; específicamente, en el artículo 64 establece: "Será obligación de los padres o encargados matricular a las personas menores de edad en el centro de enseñanza que corresponda, exigirles la asistencia regular y participar activamente en el proceso educativo" (Costa Rica, Ministerio de Educación Pública, 2005a, p. 46).

Asimismo, en estos manuales de procedimientos se definen otras responsabilidades, entre las que se pueden mencionar: acudir al centro educativo y pedir una cita con el docente a cargo, conocer los servicios de apoyo del centro educativo, reunirse con los orientadores y el Comité de Apoyo o con el docente de educación especial para conocer sobre los procesos educativos de sus hijos, conocer los reglamentos educativos, darle seguimiento al programa educativo en el hogar con la guía del docente, asistir al servicio de apoyo cuando la docente lo solicite y, por último, asistir y participar en las reuniones de padres de familia.

Para cumplir con estos deberes, se debe señalar, de forma específica, a los padres que tienen hijos o hijas con necesidades educativas, que están en la obligación de ofrecer toda la información que ayude al docente y al Comité de Apoyo a completar el historial de cada estudiante, para aplicar la adecuación curricular significativa necesaria (Costa Rica, Ministerio de Educación Pública, 2005a). Sin embargo, éste no es un requisito indispensable para que la adecuación se lleve a cabo.

\section{Metodología}

El estudio realizado fue de tipo descriptivo, según el cual se da cuenta de situaciones, eventos y hechos como se presentan en la realidad (Hernández, Fernández y Baptista, 2003), por lo que la indagación permite un acercamiento a las diferentes formas de apoyo familiar que se proporcionan durante el proceso de integración educativa de los estudiantes con necesidades educativas, desde la perspectiva de los docentes, de los miembros de la familia y de los mismos estudiantes.

También se considera que la investigación realizada es de tipo exploratorio (Barrantes, 1999), porque el tema de la indagación (análisis del tipo de apoyo familiar durante el proceso de integración de estudiantes con necesidades educativas en condición de discapacidad) no ha sido estudiado o abordado con anterioridad.

Esta investigación se llevó a cabo en una institución pública de la provincia de San José denominada de atención prioritaria, por ubicarse en una zona urbana que presenta problemas 
sociales, los cuales se reflejan en la drogadicción, la delincuencia y el vandalismo en algunos miembros de la comunidad.

En el estudio, participaron 33 estudiantes con necesidades educativas, 29 padres de familia y 28 docentes de I y II ciclos.

\section{Instrumentos}

Para la recolección de la información se empleó una técnica de indagación descriptiva, como es la entrevista, técnica que persigue conocer la opinión de los sujetos mediante la conversación dirigida sobre un tema de interés, con el fin de obtener la mayor cantidad de información (Barrantes, 1999). Ésta fue aplicada por las responsables del estudio, con el fin de develar los significados, las construcciones de los participantes y las percepciones de los docentes, de los estudiantes y de los miembros de la familia, sobre el apoyo familiar durante el proceso de integración educativa.

Para la validación de la entrevista se empleó el criterio de jueces, para lo cual se seleccionaron ocho profesionales en educación con grado académico de Licenciatura, Maestría y Doctorado. Luego, se efectúo una prueba piloto con docentes de I y II ciclos y con un grupo de padres de familia que tienen hijos con necesidades educativas, los cuales no formaban parte de la investigación.

La entrevista dirigida a los estudiantes constaba de cuatro preguntas abiertas relacionadas con el tipo de apoyo que les proporcionan los miembros de las familias en su estudio, con la persona responsable de ayudarle en el hogar y con los aspectos o situaciones que agradan y desagradan durante el apoyo familiar.

La entrevista dirigida a los docentes constaba de siete preguntas abiertas relacionadas con el concepto de apoyo familiar durante el proceso de integración educativa, con los tipos de apoyo que proporcionan los miembros de la familia a los estudiantes, con la forma en que los miembros de la familia coordinan con la docente el apoyo y con el seguimiento a los estudiantes en su estudio.

La entrevista dirigida a los miembros de familia constó de siete preguntas abiertas que trataban sobre el concepto de apoyo familiar, la importancia de este apoyo, los tipos de apoyo que proporcionan los miembros de las familias, las personas responsables de apoyar al estudiante en el hogar y la forma en que se coordinan con la docente de I o II ciclos.

Para el desarrollo de la investigación se gestionó el permiso respectivo ante el director de la institución educativa; se solicitó el consentimiento informado a cada una de las personas participantes mediante una nota escrita, en la cual se le informó sobre los objetivos de la investigación y, además, se le garantizó la confiabilidad de los datos personales.

La realización del estudio se llevó a cabo en tres etapas. En la primera etapa, se concibió el tema por indagar, se plantearon el problema y los objetivos, se elaboraron los instrumentos, se definieron la justificación de la investigación y el cronograma de trabajo.

En la segunda, se construyó el marco teórico referencial para respaldar el tema de investigación, para lo cual se efectuaron tres tareas, a saber: la revisión de la literatura, la recopilación de la información pertinente y la construcción del marco teórico.

En la tercera, se efectuaron los procedimientos de recolección de información y de análisis de los datos, para lo cual, se realizaron los siguientes procesos: la validación de los instrumentos, la recolección de la información, la tabulación de los datos en matrices temáticas, la elaboración de figuras y tablas con los resultados, así como, el análisis de las categorías generadas sobre la temática. Por último, se llevó a cabo la redacción del informe final. 


\section{Resultados}

Con el fin de responder a los objetivos de la investigación se presenta a continuación la información más relevante (opiniones de los participantes según la frecuencia absoluta y los porcentajes) organizada en tablas y figuras.

Tabla 1

Concepto de apoyo familiar que tienen los docentes de I y II ciclos en el proceso de integración escolar. Frecuencias absoluta y relativa

\begin{tabular}{|l|c|c|}
\hline \multicolumn{1}{|c|}{ Concepto } & $\mathrm{f}$ & $\%$ \\
\hline $\begin{array}{l}\text { Ayuda que se brinda a estudiantes por medio de una comunicación cons- } \\
\text { tante. }\end{array}$ & 16 & 57 \\
\hline $\begin{array}{l}\text { Interés, disposición y compromiso de los padres de familia en el proceso } \\
\text { educativo de los niños. }\end{array}$ & 7 & 25 \\
\hline $\begin{array}{l}\text { Requisito fundamental para la aplicación de la adecuación curricular en el } \\
\text { proceso de enseñanza aprendizaje. }\end{array}$ & 4 & 14 \\
\hline \begin{tabular}{l} 
Concepción de que el apoyo en el hogar refleja buenas calificaciones. \\
\hline
\end{tabular}
\end{tabular}

Nota: Elaborado por Fontana, A., Alvarado, A., Angulo, M., Marín, E y Quirós, M. en el año 2008. Aplicado a 28 docentes.

Al analizar los datos en forma global y descendente, se aprecia que la mayoría de los docentes de I y II ciclos consideran que el apoyo familiar es la ayuda que se le brinda al estudiante en el hogar para hacer una tarea o estudiar, y que la comunicación constante con el hogar favorece el establecimiento de diferentes formas de apoyo escolar.

Una parte significativa de los docentes opinan que las muestras de interés, disposición y compromiso de los padres y madres en el proceso de integración escolar, es otra forma de apoyo familiar.

Un grupo menor considera que el apoyo familiar debería ser un requisito fundamental para la aplicación de las adecuaciones curriculares en los procesos de enseñanza y de aprendizaje. Sólo una docente parte de la concepción de que "Un niño con buenas calificaciones tiene apoyo familiar".

De los resultados anteriores, se infiere que los docentes de I y II ciclos consideran que el apoyo familiar es la ayuda que se le proporciona al estudiante en el hogar para realizar diversas actividades escolares, y que este apoyo se fortalece cuando los padres de familia muestran interés, disposición y compromiso en la formación de sus hijos.

Si se comparan las apreciaciones de los docentes de I y II ciclos con la opinión de los padres de familia de los estudiantes con necesidades educativas en condición de discapacidad, se pueden apreciar ciertas coincidencias, porque éstos consideran que el apoyo familiar es la ayuda que se proporciona en el hogar, es decir, el cuidado y la atención de las necesidades que presentan sus hijos en las actividades escolares. 
En la Tabla 2, se muestran las razones que dan los docentes de I y II ciclos sobre la importancia del apoyo familiar durante el proceso de integración educativa.

Tabla 2

Razones que dan los docentes de I y II ciclos sobre la importancia del apoyo familiar en el proceso de integración escolar. Frecuencias absoluta y relativa

\begin{tabular}{|l|c|c|}
\hline \multicolumn{1}{|c|}{ Razones } & $\mathrm{f}$ & $\%$ \\
\hline $\begin{array}{l}\text { Propicia en los padres de familia interés, conocimientos y una buena } \\
\text { comunicación durante los procesos de enseñanza y de aprendizaje. }\end{array}$ & 11 & 39 \\
\hline $\begin{array}{l}\text { Favorece el proceso de enseñanza, la labor del docente y las actitudes posi- } \\
\text { tivas hacia la escuela. }\end{array}$ & 9 & 32 \\
\hline $\begin{array}{l}\text { Permite que el estudiante logre conocimientos de sí mismo, mejore su } \\
\text { autoestima, tenga mayor seguridad y logre progresar en su estudio. }\end{array}$ & 07 & 25 \\
\hline \begin{tabular}{l} 
No responde. \\
\hline
\end{tabular}
\end{tabular}

Nota: Elaborado por Fontana, A., Alvarado, A., Angulo, M., Marín, E y Quirós, M. en el año 2008. Aplicado a 28 docentes.

Puede apreciarse que una cantidad muy significativa de los docentes de I y II ciclos consideran importante el apoyo familiar en el proceso de integración escolar, porque propicia la participación, el interés, el conocimiento y la comunicación de los miembros de la familia en los procesos de enseñanza y de aprendizaje de sus hijos.

Una parte significativa de docentes menciona que este apoyo favorece la labor docente y el desarrollo de actitudes positivas hacia la institución, aspectos que, a su vez, generan mayor participación de la familia en el proceso educativo.

Un grupo menor de docentes opina que el apoyo familiar durante el proceso de integración escolar fortalece la autoestima, la seguridad y el autoconocimiento de los estudiantes, promoviendo, de esta forma, un mejor desarrollo personal, y el aprovechamiento de las oportunidades del contexto escolar.

De los resultados anteriores, puede notarse que los docentes consideran importante el apoyo de la familia durante el proceso de integración educativa, porque favorece la comunicación con los miembros de la familia y el desarrollo de actitudes positivas hacia la institución; asimismo, fortalece la autoestima, la seguridad y el autoconcepto de los estudiantes.

Al respecto, la Ley 7600 (Costa Rica. Asamblea Legislativa, 1996) establece en su artículo 20 que los padres y las madres de familia de estudiantes con discapacidad pueden participar en la selección, la ubicación, la organización y la evaluación del proceso educativo que se lleva a cabo con sus hijos. Por tanto, la participación de la familia en la formación de sus hijos es un derecho, un deber, una responsabilidad que se debe ejercer para que los estudiantes puedan disfrutar plenamente de la educación en su centro educativo. 
Por su parte, los padres de familia también consideran importante el apoyo que se proporciona en el hogar, porque sus hijos cumplen con las labores escolares, mejoran su disciplina en el estudio y aprenden los temas que más se les dificulta; y porque estos aspectos favorecen su integración y su adaptación en el contexto educativo. Además, esta ayuda permite a los miembros de la familia mantenerse informados sobre el tipo de apoyo que proporciona la docente de I y II ciclos a sus hijos.

Cabe resaltar que este aspecto ha sido tratado por el Ministerio de Educación Pública (2005a) al plantear que la participación de la familia es una de las condiciones decisivas para obtener éxito en la formación de los estudiantes.

En la Tabla 3 se recopilan las diferentes formas en que la familia proporciona apoyo a sus hijos, según el criterio del los docentes de I y II ciclos.

Tabla 3

Tipos de apoyo que proporciona la familia durante el proceso de integración escolar, según la opinión de los docentes de I y II ciclos. Frecuencias absoluta y relativa

\begin{tabular}{|l|c|c|}
\hline \multicolumn{1}{|c|}{ Tipos de apoyo } & f & $\%$ \\
\hline $\begin{array}{l}\text { Apoyo educativo mediante la realización de tareas y trabajos extra-clase, } \\
\text { revisión de cuadernos y la respuesta a preguntas. }\end{array}$ & 16 & 57 \\
\hline $\begin{array}{l}\text { Apoyo emocional, motivación, atención y vigilancia del comportamiento } \\
\text { y límites del niño. }\end{array}$ & 12 & 42 \\
\hline Comunicación constante con el docente y asistencia a reuniones. & 10 & 35 \\
\hline $\begin{array}{l}\text { Control de la puntualidad y responsabilidad del estudiante para evitar el } \\
\text { ausentismo. }\end{array}$ & 7 & 25 \\
\hline $\begin{array}{l}\text { Apoyo económico y con material, mediante la compra de libros, cuader- } \\
\text { nos, lápices, goma y otros. }\end{array}$ & 04 & 014 \\
\hline Atención de las necesidades de salud y nutrición. & 2 & 7 \\
\hline Ninguno. & 1 & 4 \\
\hline
\end{tabular}

Nota: Elaborado por Fontana, A., Alvarado, A., Angulo, M., Marín, E y Quirós, M. en el año 2008. Aplicado a 28 docentes. La suma de los porcentajes no corresponde a un 100\%, pues los entrevistados escogieron varias opciones.

Al analizar los datos, puede apreciarse que que la mayoría de los docentes de I y II ciclos considera que el apoyo educativo que más brinda la familia durante el proceso de integración se lleva a cabo mediante la realización de tareas y trabajos extra clase, revisión de cuadernos y la respuesta a cuestionarios o preguntas. 
Una gran parte de docentes opina que la familia proporciona apoyo emocional, motivación y vigilancia del comportamiento de sus hijos.

Asimismo, un número significativo de docentes expresa que los miembros de la familia apoyan a sus hijos mediante la asistencia a reuniones y la comunicación con el docente; un grupo más pequeño menciona que el control de la puntualidad y la responsabilidad del estudiante es una forma de apoyo familiar.

Un grupo menor de docentes considera que los padres de familia proporcionan apoyo económico y material, así como, la atención a las necesidades de salud y nutrición.

Sólo una docente opina que los padres de familia no ofrecen ningún tipo de apoyo a sus hijos en el proceso de formación.

De los datos anteriores, puede afirmarse que los docentes de I y II ciclos consideran que las familias proporcionan diferentes tipos de apoyo a sus hijos durante el proceso de integración escolar, los cuales se relacionan, principalmente, con aspectos educativos, emocionales y económicos. Asimismo, opinan que la comunicación y la coordinación constantes con los miembros de la familia favorecen estos tipos de apoyo en los procesos de enseñanza y de aprendizaje. Estos resultados son congruentes con lo que expresa Heward (1997), quien indica que la comunicación constante con la familia permite sobrellevar cualquier situación que se presente en la atención educativa de los estudiantes y que, a la vez, fortalece los procesos de formación.

Los padres de familia coinciden con los docentes de I y II ciclos al indicar que los tipos de apoyo familiar que brindan a sus hijos son, principalmente, para realizar las tareas, concluir los trabajos pendientes y comprar materiales didácticos, como los libros y cuadernos. Además, indican que este apoyo se fortalece si se les dedica tiempo y se les tiene paciencia, así como si se establecen distintas formas de comunicación con la docente de I y II ciclos.

Lo anterior corrobora lo que plantean Sánchez y Torres (2002) cuando indican la importancia de la permanencia y la intervención de los miembros de la familia en la formación de sus hijos, pues eso favorece tanto el logro de los objetivos y contenidos propuestos, como la interrelación y la comunicación entre sus miembros, particularmente, las relaciones padre-hijo, madre-hijo o hermano-hermano.

La Tabla 4 muestra las formas de apoyo que proporciona la familia en el proceso de integración educativa, según la opinión de los estudiantes.

Tabla 4

Formas de apoyo que proporciona la familia al estudiante con adecuación curricular significativa durante el proceso de integración escolar, según la opinión de los estudiantes. Frecuencias absoluta y relativa

\begin{tabular}{|l|c|c|}
\hline \multicolumn{1}{|c|}{ Formas } & $\mathrm{f}$ & $\%$ \\
\hline Realizar prácticas de lecturas y operaciones básicas (suma y resta). & 16 & 48 \\
\hline Realizar tareas y contestar cuestionarios. & 11 & 34 \\
\hline Comprar materiales. & 3 & 9 \\
\hline Estudiar sin ayuda. & 3 & 9 \\
\hline
\end{tabular}

Nota: Elaborado por Fontana, A., Alvarado, A., Angulo, M., Marín, E y Quirós, M. en el año 2008. Aplicado a 33 estudiantes. 
Al analizar los datos de forma global se aprecia que una gran cantidad de los estudiantes opina que el apoyo que se les brinda en sus hogares consiste en la realización de prácticas de lectura y operaciones básicas.

Una parte muy significativa de estudiantes menciona que este apoyo se centra en la realización de tareas y elaboración de cuestionarios.

Un grupo menor expresa que el apoyo que se le brinda en el hogar consiste en la compra de materiales. Es importante resaltar que una cantidad similar de estudiantes opina que estudian sin ningún tipo de ayuda.

De los resultados anteriores, se puede afirmar que los estudiantes reciben diferentes tipos de apoyo por parte del núcleo familiar, los cuales se centran, principalmente, en la realización de prácticas en las áreas académicas de Español y Matemática. Sólo un grupo muy pequeño estudia sin ayuda.

Al comparar las opiniones expresadas por los docentes y los padres de familia con las apreciaciones de los estudiantes se aprecia coincidencia entre ellas, principalmente, con el tipo de apoyo que reciben en su estudio (realización de tareas, trabajos extraclase, contestar cuestionarios).

En la Figura 1 se muestra la razón principal del por qué a una mayoría de los estudiantes no les agrada que les brinden apoyo en el estudio, en su núcleo familiar.

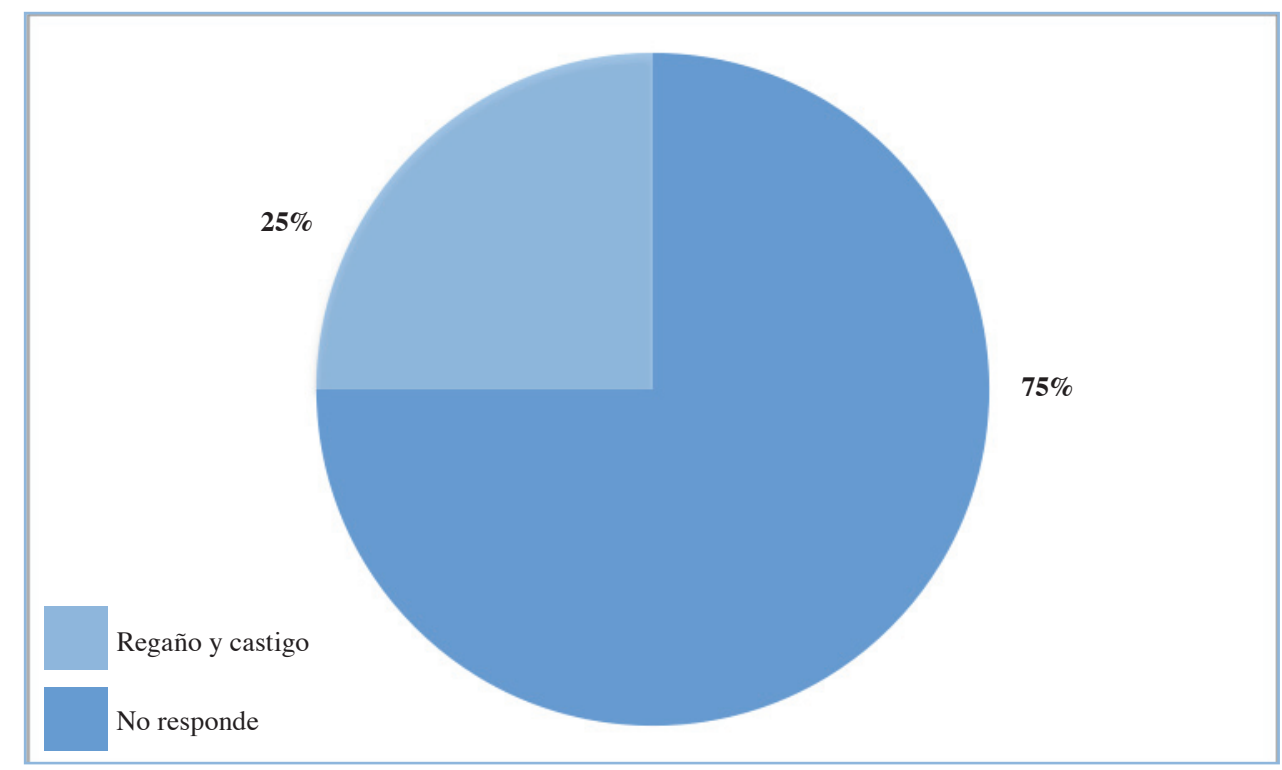

Figura 1. Razones que expresan los estudiantes con adecuación curricular significativa del por qué no les agrada que les ayuden a estudiar en el hogar.

Nota: Elaborado por Fontana, A., Alvarado, A., Angulo, M., Marín, E y Quirós, M. en el año 2008. Aplicado a 33 estudiantes.

Un aspecto significativo de resaltar, es que la mayoría de los estudiantes indicó que no les agrada el apoyo que se le proporciona en el hogar, porque éste se relaciona con el regaño o el castigo, razón por la cual, prefieren realizar sus labores escolares solos. No obstante, sólo una parte muy pequeña de estos estudiantes trabaja sin ayuda de la familia.

En la comparación entre los resultados expuestos en la Figura 1 con lo expresado por los docentes y los padres de familia anteriormente en las Tablas 1, 2 y 3, se muestra un contraste 
muy significativo, porque mientras para una mayoría de docentes de I y II ciclos y de padres de familia que participaron en el estudio, los diferentes tipos de apoyo familiar son importantes en la formación educativa de los estudiantes con necesidades educativas en condición de discapacidad, para la mayoría de estos estudiantes ese apoyo se asocia con sanciones y castigos, lo cual genera sentimientos de malestar que inciden en la disposición y el interés de ellos hacia el estudio.

Por lo anterior, es importante reflexionar en el contexto familiar y educativo el trato, las actitudes y las formas de comunicación e interrelación que se presentan entre las personas responsables y los estudiantes en el momento de proporcionar el apoyo en el hogar. Asimismo, es relevante que se proporcione asesoría a los miembros de la familia sobre las estrategias apropiadas para apoyar a sus hijos en el estudio.

La Tabla 5 muestra a la persona o a las personas de la familia o allegados, responsables de apoyar a al estudiante durante el proceso de integración educativa.

Tabla 5

Miembro de la familia o alllegado que proporciona el apoyo al estudiante con adecuación curricular significativa durante el proceso de integración, según la opinión de los estudiantes. Frecuencias absoluta y relativa

\begin{tabular}{|l|c|c|}
\hline \multicolumn{1}{|c|}{ Personas } & $\mathrm{f}$ & $\%$ \\
\hline Hermana mayor. & 10 & 30 \\
\hline Mamá. & 7 & 21 \\
\hline Otras personas (profesora de inglés, vecinos y quienes los cuidan). & 3 & 10 \\
\hline Mamá, papá y hermanos. & 2 & 6 \\
\hline Tío. & 2 & 6 \\
\hline Primo. & 1 & 3 \\
\hline Papá. & 1 & 3 \\
\hline Padrastro. & 1 & 3 \\
\hline Ninguna persona. & 6 & 18 \\
\hline
\end{tabular}

Nota: Elaborado por Fontana, A., Alvarado, A., Angulo, M., Marín, E y Quirós, M. en el año 2008. Aplicado a 33 estudiantes.

En la Tabla 5, puede observarse que a la mayoría de los estudiantes les proporciona ayuda su hermana mayor y su madre.

A una parte menor de los estudiantes, le brindan ayuda otras personas como la profesora de inglés, la vecina y las personas que los cuidan. Un grupo aún menor de estudiantes recibe apoyo de su tío, primo, padre y padrastro.

Es importante, resaltar que un número significativo de estudiantes no recibe ningún tipo de apoyo.

De los datos anteriores, puede afirmarse que diferentes miembros de la familia le proporcionan apoyo al estudiante en el estudio, sobre todo, la hermana mayor y la madre, quienes se encargan de ayudarlos y cuidarlos en sus labores escolares, aunque esta es una función básica 
de todos los miembros de la familia, para potenciar al máximo el desarrollo de habilidades y destrezas de los hijos.

Lo anterior, pone en evidencia que en la cultura familiar todavía prevalecen patrones tradicionales de crianza de los hijos, porque se delega la función educativa a la madre y a la hermana mayor, lo que pone en evidencia, tal como lo expresa Campabadall (2001), que la distribución de las tareas domésticas y responsabilidades sociales se mantienen recargadas en la mujer actual, con el legado cultural (la maternidad y la familia) y el mandato social de cambio (incorporación al trabajo profesional).

Otro aspecto que se debe resaltar, es que un grupo de estudiantes manifiesta que personas fuera del núcleo familiar le proporcionan apoyo en el estudio, tales como, profesores, vecinos y las personas que los cuidan. Éste es un aspecto digno de retomar, porque pone de manifiesto que algunas familias establecen redes de apoyo con personas fuera del núcleo familiar, tales como, docente y miembros de la comunidad.

A continuación, se presentan las distintas formas en que la familia solicita apoyo a la docente de I y II ciclos durante el proceso de integración educativa.

Tabla 6

Formas en que la familia solicita ayuda a los docentes de I y II ciclos durante el proceso de integración escolar. Frecuencias absoluta y relativa

\begin{tabular}{|l|c|c|}
\hline \multicolumn{1}{|c|}{ Formas } & f & $\%$ \\
\hline No solicitan ayuda. & 17 & 60 \\
\hline Entrevistas para dialogar sobre el proceso de aprendizaje de sus hijos. & 5 & 18 \\
\hline Reuniones de padres de familia establecidas por la escuela. & 2 & 7 \\
\hline $\begin{array}{l}\text { Preguntas orales sobre la materia y los libros de apoyo que se pueden uti- } \\
\text { lizar durante el proceso de aprendizaje. }\end{array}$ & 02 & 07 \\
\hline Mensajes al hogar por medio del libro de comunicaciones. & 1 & 4 \\
\hline La forma de solicitud varía según el nivel de escolaridad de la familia. & 1 & 4 \\
\hline
\end{tabular}

Nota: Elaborado por Fontana, A., Alvarado, A., Angulo, M., Marín, E y Quirós, M. en el año 2008. Aplicado a 28 docentes.

En la Tabla 6, se aprecia que la mayoría de docentes de I y II ciclos opinan que los padres y madres de familia no les solicitan ayuda para apoyar a sus hijos.

No obstante, otro grupo de docentes consideran que los padres de familia solicitan apoyo mediante entrevistas para dialogar sobre el proceso de aprendizaje de sus hijos, en reuniones de grupo y con preguntas orales, sobre la materia y los libros de apoyo que requieren. Sólo una docente opina que la familia emplea el libro de comunicaciones al hogar para solicitar ayuda.

Es importante resaltar, que una docente considera que el nivel educativo de la familia determina la forma en que ésta solicita apoyo.

Según los resultados obtenidos, puede afirmarse que la mayoría de los padres de familia no solicitan apoyo a los docentes de I y II ciclos para ayudar a sus hijos en el hogar, situación 
que evidencia la falta de comunicación y de coordinación entre los miembros de la familia y los docentes de I y II ciclos; este aspecto no favorece el proceso de integración escolar. Sin embargo, los padres de familia que sí solicitan apoyo emplean diferentes formas de comunicación, tales como entrevistas, conversaciones, participación en las reuniones de grupo y el libro de comunicaciones, aspectos que favorecen el proceso de formación de los estudiantes.

En este sentido, la Ley 7600 (Costa Rica. Asamblea Legislativa, 1996) en su artículo 11 indica que los miembros de la familia de las personas con discapacidad deben apoyar su desarrollo integral, lo que pone en evidencia la necesidad del apoyo continuo y constante del núcleo familiar, para enriquecer el proceso educativo.

Por su parte, los docentes de I y II ciclos indican que utilizan diferentes estrategias de coordinación con la familia. En la Tabla 7 se muestran las formas empleadas.

Tabla 7

Formas en que el docente de I y II ciclos coordina el apoyo familiar de los estudiantes con adecuación curricular significativa durante el proceso de integración escolar. Frecuencias absoluta y relativa

\begin{tabular}{|l|r|c|}
\hline \multicolumn{1}{|c|}{ Forma } & f & $\%$ \\
\hline Comunicación constante mediante recados y llamadas telefónicas al hogar. & 14 & 50 \\
\hline Realización de reuniones y talleres con padres de familia. & 9 & 32 \\
\hline Visitas al hogar. & 2 & 7 \\
\hline Incorporación de los padres de familia en las actividades escolares. & 2 & 7 \\
\hline Motivación constante al estudiante. & 1 & 4 \\
\hline
\end{tabular}

Nota: Elaborado por Fontana, A., Alvarado, A., Angulo, M., Marín, E y Quirós, M. en el año 2008. Aplicado a 28 docentes.

Al analizar los datos de forma global y descendente puede observarse que la mayoría de los docentes de I y II ciclos emplean la comunicación por medio de recados y llamadas telefónicas con los familiares. familia.

Una parte amplia de docentes indica que realiza reuniones y talleres con los padres de

Un grupo pequeño menciona que realiza visitas al hogar e incorpora a los padres de familia en las actividades escolares. Sólo una docente expresa que motiva a los estudiantes para que los padres de familia proporcionen ayuda en el estudio.

De estos resultados, puede afirmarse que los docentes de I y II ciclos realizan diferentes acciones para comunicarse con los miembros de la familia, con el fin de promover el apoyo en el estudio. Estas acciones coinciden con las indicadas por los padres de familia y repercuten, favorablemente, con el proceso de integración educativa de los estudiantes con necesidades educativas en formas de comunicación con el docente que atiende a sus hijos, tendrán la oportunidad de apoyar a los alumnos en el estudio según las necesidades que presentan, así como de conocer las responsabilidades que tiene que cumplir. De esta manera, la comunicación y la interrelación entre los miembros de la familia y el personal docente del centro educativo se verán fortalecidas. 


\section{Conclusiones}

De acuerdo con el análisis de los datos obtenidos pudieron extraerse las siguientes conclusiones:

1. Se determina que los docentes de I y II ciclo que participaron en este estudio, consideran que el apoyo familiar consiste en la ayuda que se le proporciona al estudiante en el hogar, para realizar diversas actividades escolares y que el interés, la disposición, el compromiso de los padres de familia o encargados, así como la comunicación constante con ellos favorecen el establecimiento de nuevas formas de apoyo familiar durante el proceso de integración educativa de estudiantes con necesidades educativas en condición de discapacidad, las cuales, se constituyen en el vínculo entre los dos entornos vitales más importantes en su formación educativa, como lo son el núcleo familiar y la comunidad del centro educativo.

2. Se establece que el apoyo familiar durante el proceso de integración educativa es importante para el desarrollo integral de los estudiantes, porque tiene repercusiones tanto en el ámbito educativo como en el ámbito familiar y personal de los estudiantes, ya que mediante estos apoyos los estudiantes alcanzan los objetivos y los contenidos propuestos en los procesos de enseñanza y de aprendizaje, se promueven distintas formas de comunicación e interrelación entre los miembros de la familia y el personal docente y administrativo del centro educativo; asimismo, se mejora su autoestima, la seguridad y el conocimiento de sí mismo, lo que posibilita su inclusión social y laboral.

3. Se demuestra que los tipos de apoyo que brindan los miembros de la familia a los estudiantes con necesidades educativas en condición de discapacidad durante el proceso de integración educativa se centran, principalmente, en aspectos educativos (realización de tareas y trabajos) y provisión de materiales didácticos (compra de libros y útiles escolares). Estos tipos de apoyo se fortalecen si los miembros de la familia le dedican tiempo, muestran paciencia y tolerancia durante el apoyo y mantienen distintas formas de comunicación con el docente de I y II ciclos, los cuales van a facilitar el progreso académico de los estudiantes y su participación en las distintas actividades educativas.

4. Se determina que a la mayoría de los estudiantes con necesidades educativas en condición de discapacidad les desagrada que les brinden ayuda en el hogar para realizar las labores escolares, porque los regañan y los castigan durante este apoyo. Sin embargo, al grupo de estudiantes que les agrada el apoyo que les proporcionan los miembros de su familia se debe, principalmente, porque logran comprender y entender los contenidos estudiados y, de esta forma, obtienen mejores calificaciones.

5. Se constata que los docentes de I y II ciclos utilizan diversas acciones y estrategias para comunicarse con los padres de familia; se destaca el envío de recados, reuniones, llamadas telefónicas y talleres. No obstante, la mayoría de los miembros de la familia de los estudiantes no solicita apoyo para ayudar a sus hijos en el hogar, situación que evidencia una falta de comunicación y coordinación entre ambas partes, aspecto que incide en la calidad y en el tipo de apoyo familiar durante el proceso de integración educativa.

6. Se determina que los padres de familia solicitan apoyo tanto a los docentes de I y II ciclos como a las docentes de Educación Especial, utilizando distintos medios, tales como: la entrevista, la participación en reuniones y el uso del libro de comunicaciones.

7. Se evidencia que los padres de familia que solicitan apoyo a la docente de Educación Especial tienen interés por conocer el avance de sus hijos en el proceso de aprendizaje 
y, al mismo tiempo, cerciorarse del comportamiento y del seguimiento de las necesidades educativas que presentan sus hijos para proporcionarles la ayuda requerida, aspecto esencial para el progreso académico del estudiante en el contexto educativo.

8. Se confirma que la responsabilidad de brindar apoyo en el hogar a los estudiantes con necesidades educativas en condición de discapacidad recae, principalmente, en la hermana mayor y la madre, quienes son las responsables de ayudarlos en las labores escolares, lo que evidencia que en la cultura familiar aún prevalece una visión centrada en los patrones tradicionales de crianza, la que delega la función educativa a la mujer.

\section{Referencias bibliográficas}

Arés, P. (1997a). Psicología de la familia. Taller internacional de familia. La Habana, Cuba: Facultad de Psicología, Universidad de la Habana.

Arés, P. (1997b). La familia una mirada al futuro. Taller internacional de familia. La Habana, Cuba: Facultad de Psicología, Universidad de la Habana.

Barrantes, R. (1999). Investigación. Un camino al conocimiento. Un enfoque cualitativo y cuantitativo. San José, Costa Rica: Editorial Universidad Estatal a Distancia.

Campabadal, M. (2001). El niño con discapacidad y su entorno. San José, Costa Rica: Editorial Universidad Estatal a Distancia.

Costa Rica. Asamblea Legislativa. (1996). Ley 7600. Igualdad de Oportunidades para la Personas con Discapacidad. San José, Costa Rica: Imprenta Nacional.

Costa Rica. Asamblea Legislativa. (2008). Ley 8661. Convención sobre los derechos de las Personas con Discapacidad y su protocolo. San José, Costa Rica: Imprenta Nacional.

Costa Rica. Ministerio de Educación Pública. (2005a). Normas y procedimientos para el manejo técnico administrativo de los servicios educativos para estudiantes con retardo mental. San José, Costa Rica: Centro Nacional de Recursos para la Educación Inclusiva.

Costa Rica. Ministerio de Educación pública. (2005b). Políticas, Normativa y Procedimientos para el acceso a la Educación de los Estudiantes con Necesidades Educativas Especiales. San José, Costa Rica: Centro Nacional de Recursos para la Educación Inclusiva.

Flores, S. \& Rodríguez, C. (2000). Características de la familia uniparental y las relaciones sociales de los niños y las niñas entre 4 y 8 años. Miembros de estas familias y que asisten a la guardería del Hospicio de Huérfanos de San José. Tesina para optar por el grado de Bachillerato en Trabajo Social no publicada. San José, Universidad de Costa Rica.

Hernández Sampieri, R., Fernández Collado, C. \& Baptista Lucio, P. (2003). Metodología de la investigación. ( $3^{\mathrm{a}}$ ed.). México: Editorial McGraw-Hill. 
Heward, W. (1997). Niños excepcionales: una introducción a la Educación Especial. Madrid, España: Prentice Hall.

Organización Panamericana de la Salud. (2004). La discapacidad en Costa Rica: situación actual y perspectivas. San José, Costa Rica: Organización Panamericana de la Salud.

Sánchez Palomino, A. \& Torres González, J. (2000). Educación Especial. Centros educativos y profesorados ante la diversidad. Madrid, España: Ediciones Pirámide. 